\title{
Cost Edge-Coloring of a Cactus
}

\author{
Zhiqian Ye'1, Yiming Lí2 ${ }^{2}$ Huiqiang Lu', Xiao Zhou ${ }^{4}$ \\ ${ }^{1}$ Zhejiang University, Hanzhou, China \\ ${ }^{2}$ Wenzhou University, Wenzhou, China \\ ${ }^{3}$ Zhejiang University of Technology, Hangzhou, China \\ ${ }^{4}$ Tohoku University, Sendai, Japan \\ Email: yezhiqian@zju.edu.cn, ymli@wzu.edu.cn, lhq@zjut.edu.cn, zhou@ecei.tohoku.ac.jp
}

Received 11 August 2015; accepted 15 October 2015; published 22 October 2015

\begin{abstract}
Let $C$ be a set of colors, and let $\omega(c)$ be an integer cost assigned to a color $c$ in $C$. An edge-coloring of a graph $G=(V, E)$ is assigning a color in $C$ to each edge $e \in E$ so that any two edges having end-vertex in common have different colors. The cost $\omega(f)$ of an edge-coloring $f$ of $G$ is the sum of costs $\omega(f(e))$ of colors $f(e)$ assigned to all edges $e$ in $G$. An edge-coloring $f$ of $G$ is optimal if $\omega(f)$ is minimum among all edge-colorings of $G$. A cactus is a connected graph in which every block is either an edge or a cycle. In this paper, we give an algorithm to find an optimal edgecoloring of a cactus in polynomial time. In our best knowledge, this is the first polynomial-time algorithm to find an optimal edge-coloring of a cactus.
\end{abstract}

\section{Keywords}

Cactus, Cost Edge-Coloring, Minimum Cost Maximum Flow Problem

\section{Introduction}

Let $G=(V, E)$ be a graph with vertex set $V$ and edge set $E$, and let $C$ be a set of colors. An edge-coloring of $G$ is to color all the edges in $E$ so that any two adjacent edges are colored with different colors in $C$. The minimum number of colors required for edge-colorings of $G$ is called the chromatic index, and is denoted by $\chi^{\prime}(G)$. It is well-known that $\Delta(G) \leq \chi^{\prime}(G) \leq \Delta(G)+1$ for every simple graph $G$ and that $\chi^{\prime}(G)=\Delta(G)$ for every bipartite (multi)graph $G$, where $\Delta(G)$ is the maximum degree of $G$ [1]. The ordinary edge-coloring problem is to compute the chromatic index $\chi^{\prime}(G)$ of a given graph $G$ and find an edge-coloring of $G$ using $\chi^{\prime}(G)$ colors. Let $\omega$ be a cost function which assigns an integer $\omega(c)$ to each color $c \in C$, then the cost edge-coloring problem is to find an optimal edge-coloring of $G$, that is, an edge-coloring $f$ such that $\sum_{e \in E} \omega(f(e))$ is minimum among all edge-colorings of $G$. An optimal edge-coloring does not always use the minimum number $\chi^{\prime}(G)$ of colors. For example, suppose that $\omega\left(c_{1}\right)=1$ and $\omega\left(c_{i}\right)=2$ for each index $i \geq 2$, then the graph $G$ with $\chi^{\prime}(G)=3$ in Figure 1(a) can be uniquely colored with the three cheapest colors $c_{1}, c_{2}$ and $c_{3}$ as in Figure 1(a), but this edge-coloring is not optimal; an optimal edge-coloring of $G$ uses the four cheapest colors $c_{1}, c_{2}, c_{3}$ and $c_{4}$, as illustrated in Figure 1(b). However, every simple graph $G$ has an edge-coloring 


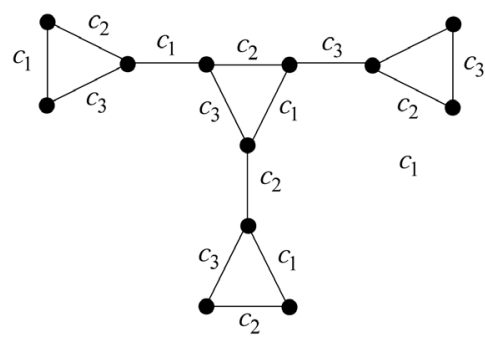

(a)

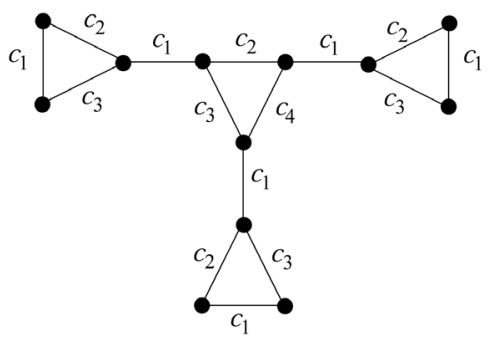

(b)

Figure 1. (a) An edge-coloring using $\chi^{\prime}(G)=3$ colors, and (b) an optimal edge-coloring using $\chi^{\prime}(G)+1=4$ colors, where $\omega\left(c_{1}\right)=1$ and $\omega\left(c_{2}\right)=\omega\left(c_{3}\right)=\omega\left(c_{4}\right)=2$.

using $\Delta(G)$ or $\Delta(G)+1$ colors [2] [3]. The edge-chromatic sum problem, introduced by Giaro and Kubale [4], is merely the cost edge-coloring problem for the special case where $\omega\left(c_{i}\right)=i$ for each integer $i \geq 1$.

The cost edge-coloring problem has a natural application for scheduling [5]. Consider the scheduling of biprocessor tasks of unit execution time on dedicated machines. An example of such tasks is the file transfer problem in a computer network in which each file engages two corresponding nodes, sender and receiver, simultaneously [6]. Another example is the biprocessor diagnostic problem in which links execute concurrently the same test for a fault tolerant multiprocessor system [7]. These problems can be modeled by a graph $G$ in which machines correspond to the vertices and tasks correspond to the edges. An edge-coloring of $G$ corresponds to a schedule, where the edges colored with color $c_{i} \in C$ represent the collection of tasks that are executed in the ith time slot. Suppose that a task executed in the ith time slot takes the cost $\omega\left(c_{i}\right)$. Then the goal is to find a schedule that minimizes the total cost, and hence this corresponds to the cost edge-coloring problem.

The cost edge-coloring problem is APX-hard even for bipartite graphs [8], and hence there is no polynomialtime approximation scheme (PTAS) for the problem unless $\mathrm{P}=\mathrm{NP}$. On the other hand, Zhou and Nishizeki gave an algorithm to solve the cost edge-coloring problem for trees $T$ in time $O\left(n \Delta^{1.5} \log \left(n N_{\omega}\right)\right)$, where $n$ is the number of vertices in $T, \Delta$ is the maximum degree of $T$, and $N_{\omega}$ is the maximum absolute cost $|\omega(c)|$ of colors $c$ in $C$ [5]. The algorithm is based on a dynamic programming (DP) approach, and computes a DP table for each vertex of a given tree $T$ from the leaves to the root of $T$. In this paper, we give a polynomial-time algorithm to solve the cost edge-coloring problem for cacti. In our best knowledge, this is the first polynomialtime algorithm to find an optimal edge-coloring of a cactus.

\section{Preliminaries}

In this section, we define some basic terms.

Let $G=(V, E)$ be a graph with a set $V$ of vertices and a set $E$ of edges. We sometimes denote by $V(G)$ and $E(G)$ the vertex set and the edge set of $G$, respectively. We denote by $n(G)$ and $m(G)$, respectively, or simply by $n$ and $m$, the number of vertices and edges in $G$, that is, $n(G)=|V|$ and $m(G)=|E|$. The degree $d(v)$ of a vertex $v$ is the number of edges in $E$ incident to $v$. We denote the maximum degree of $G$ by $\Delta(G)$ or simply by $\Delta$. A cactus $G$ can be represented by an under tree $T$, which is a rooted tree. In the underlay tree $T$ of $G$, each node represents a block which is either a bridge (edge) of $G$ or an elementary cycle of $G$. If there is an edge between nodes $b_{1}$ and $b_{2}$ of $T$, then bridges or cycles of $G$ represented by $b_{1}$ and $b_{2}$ share exactly one vertex in $G$. Each node $b$ of $T$ corresponds to a subgraph $G_{b}$ of $G$ induced by all bridges and cycles represented by the nodes that are descendants of $b$ in $T$. Figure 2(a) depicts the subgraph $G_{b_{1}}$ for the child $b_{1}$ of the root $r$ of $T$. Clearly $G=G_{r}$ and $G_{b}$ is a cactus for each node $b$ of $T$. One can easily find an underlay tree $T$ of a given cactus $G$ in linear time, and hence one may assume that an underlay tree of $G$ is given. We denote by $\operatorname{ch}(b)$ the number of edges joining a node $b$ and its children in $T$. Then, $\operatorname{ch}(r)=d(r)$, and $\operatorname{ch}(b)=d(b)-1$ for every vertex $b \in V \backslash\{r\}$.

Let $C$ be a set of colors. An edge-coloring $f: E \rightarrow C$ of a graph $G$ is to color all edges of $G$ by colors in $C$ so that any two adjacent edges are colored with different colors. Let $\omega: C \rightarrow \mathbb{R}^{+}$, where $\mathbb{R}^{+}$is the set of real numbers. One may assume with loss of generality that $\omega$ is non-decreasing, that is, $\omega\left(c_{i}\right) \leq \omega\left(c_{i+1}\right)$ for any 


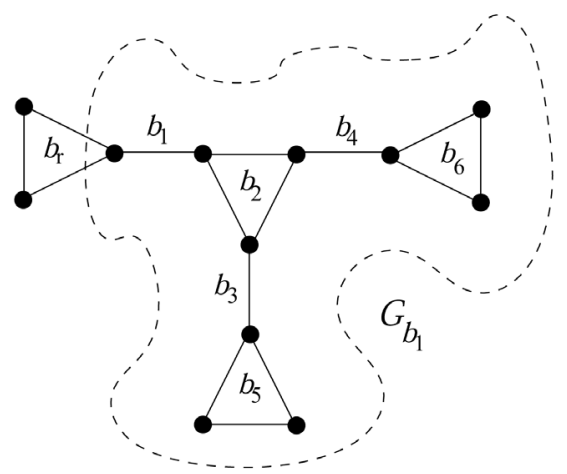

(a)

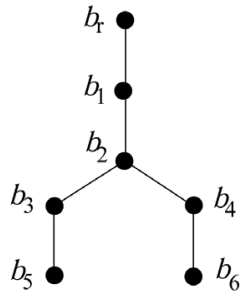

(b)

Figure 2. (a) A cactus; and (b) its under tree.

index $i, \quad 1 \leq i \leq|C|$. Since trivially any graph $G$ has an optimal edge-coloring using colors at most $2 \Delta(G)-1$, we assume for the sake of convenience that $|C|=2 \Delta(G)-1$, and we write $C=\left\{c_{1}, c_{2}, \cdots, c_{2 \Delta-1}\right\}$. The cost $\omega(f)$ of an edge-coloring $f$ of a graph $G=(V, E)$ is defined as follows:

$$
\omega(f)=\sum_{e \in E} \omega(f(e)) .
$$

An edge-coloring $f$ of $G$ is called an optimal one if $\omega(f)$ is minimum among all edge-colorings of $G$. The cost edge-coloring problem is to find an optimal edge-coloring of a given graph $G$. The cost of an optimal edge-coloring of $G$ is called the minimum cost of $G$, and is denoted by $\omega(G)$.

Let $f$ be an edge-coloring of a graph $G$. For each vertex $v$ of $G$, let $C_{f}(G, v)$ be the set of all colors that are assigned to edges incident to $v$, that is,

$$
C_{f}(G, v)=\{f(e) \mid e \text { is an edge incident to } v \text { in } G\} .
$$

We say that a color $c \in C$ is missing at $v$ if $c \notin C(f, v)$. Let $\operatorname{Miss}(f, v)$ be the set of all colors missing at $v$, that is, $\operatorname{Miss}(f, v)=C \backslash C(f, v)$.

\section{Algorithm}

In this section we prove the following theorem.

Theorem 1. An optimal edge-coloring of a cactus can be found in polynomial time.

As a proof of Theorem 1, we give a dynamic programming algorithm in the remainder of this section to compute the minimum cost $\omega(G)$ of a given cactus $G$. Our algorithm can be easily modified so that it actually finds an optimal edge-coloring $f$ of $G$ with $\omega(f)=\omega(G)$.

A dynamic programming method is a standard one to solve a combinatorial problem on graphs with treeconstruction. We also use it, and compute the minimum cost $\omega(G)$ of a cactus $G$ with an under tree $T$ by the bottom-up tree computation.

\subsection{Ideas and Definitions}

Let $b$ be a node of $T$ with its parent $b^{\prime}$, and let $v$ be the vertex on both two blocks $b$ and $b^{\prime}$. Let $b_{1}, b_{2}, \cdots, b_{\mathrm{ch}(b)}$ be the children of $b$ in $T$. Then one can observe that the minimum cost $\omega\left(G_{b}\right)$ of the subgraph $G_{b}$ rooted at $b$ cannot be computed directly from the minimum costs $\omega\left(G_{b_{j}}\right)$ of all the subgraphs $G_{b_{j}}, 1 \leq j \leq \operatorname{ch}(b)$. Our idea is to introduce a new parameter $\omega\left(G_{b}, i_{1}, i_{2}\right)$ defined for each node $b$ of $T$ and each pair of colors $c_{i_{1}}, c_{i_{2}} \in C$ as follows:

$$
\omega\left(G_{b}, i_{1}, i_{2}\right)=\min \left\{\omega(f) \mid f \text { is an edge-coloring of } G_{b} \text { and } c_{i_{1}}, c_{i_{2}} \in C(f, v)\right\} .
$$

If $G_{b}$ has no such edge-coloring we define $\omega\left(G_{b}, i_{1}, i_{2}\right)=+\infty$. Note that $\omega\left(G_{b}, i_{1}, i_{2}\right)=+\infty$ if either the block $b$ is an edge and $i_{1} \neq i_{2}$ or the block $b$ is a cycle and $i_{1}=i_{2}$. Clearly,

$$
\omega\left(G_{b}\right)=\min _{1 \leq i_{1}, i_{2} \leq 2 \Delta-1} \omega\left(G_{b}, i_{1}, i_{2}\right) .
$$


We compute the values $\omega\left(G_{b}, i_{1}, i_{2}\right)$ for all indices $i_{1}, i_{2}, \quad 1 \leq i_{1}, i_{2} \leq 2 \Delta-1$, from leaves to root $r$. Thus the DP table for each node $b$ consists of the $O\left(\Delta^{2}\right)$ values $\omega\left(G_{b}, i_{1}, i_{2}\right), \quad 1 \leq i_{1}, i_{2} \leq 2 \Delta-1$.

Our algorithm computes $\omega\left(G_{b}, i_{1}, i_{2}\right)$ for all pairs of colors $c_{i_{1}}, c_{i_{2}} \in C$ from the leaves to the root $r$ of $T$, by means of dynamic programming. Then $\omega(G)$ can be computed at the root $r$ from all the values $\omega\left(G_{r}, i_{1}, i_{2}\right)$ as follows:

$$
\omega(G)= \begin{cases}\min \left\{\omega\left(G_{r}, i, i\right) \mid c_{i} \in C\right\} & \text { if the block } r \text { is an edge; } \\ \min \left\{\omega\left(G_{r}, i_{1}, i_{2}\right) \mid c_{i_{1}}, c_{i_{2}} \in C \text { and } i_{1} \neq i_{2}\right\} & \text { if the block } r \text { is a cycle }\end{cases}
$$

and it can be computed in polynomial time. Thus the remainder problem is how to compute all the values $\omega\left(G_{b}, i_{1}, i_{2}\right)$ for each node $b \in V(T)$ of $T$ and all pairs of colors $c_{i_{1}}, c_{i_{2}} \in C$.

\subsection{Algorithm}

In this subsection, we explain how to compute all the values $\omega\left(G_{b}, i_{1}, i_{2}\right)$ for each node $b \in V(T)$ of $T$ and all pairs of colors $c_{i_{1}}, c_{i_{2}} \in C$.

\subsubsection{The Node $b$ Is a Leaf in $T$}

In this case, the block $b$ is either an edge or a cycle. Therefore we have the following two cases to consider.

Case 1: the block $b$ is an edge.

In this case, clearly

$$
\omega\left(G_{b}, i_{1}, i_{2}\right)= \begin{cases}\omega\left(c_{i_{1}}\right) & \text { if } i_{1}=i_{2} \\ +\infty & \text { if } i_{1} \neq i_{2}\end{cases}
$$

and all the values $\omega\left(G_{b}, i_{1}, i_{2}\right), \quad c_{i_{1}}, c_{i_{2}} \in C$, can be computed in time polynomial in $|C|$.

Case 2: the block $b$ is a cycle.

In this case, we describe the following algorithm to compute $\omega\left(G_{b}, i_{1}, i_{2}\right)$ in time polynomial in the size of $G_{b}$ and $|C|$.

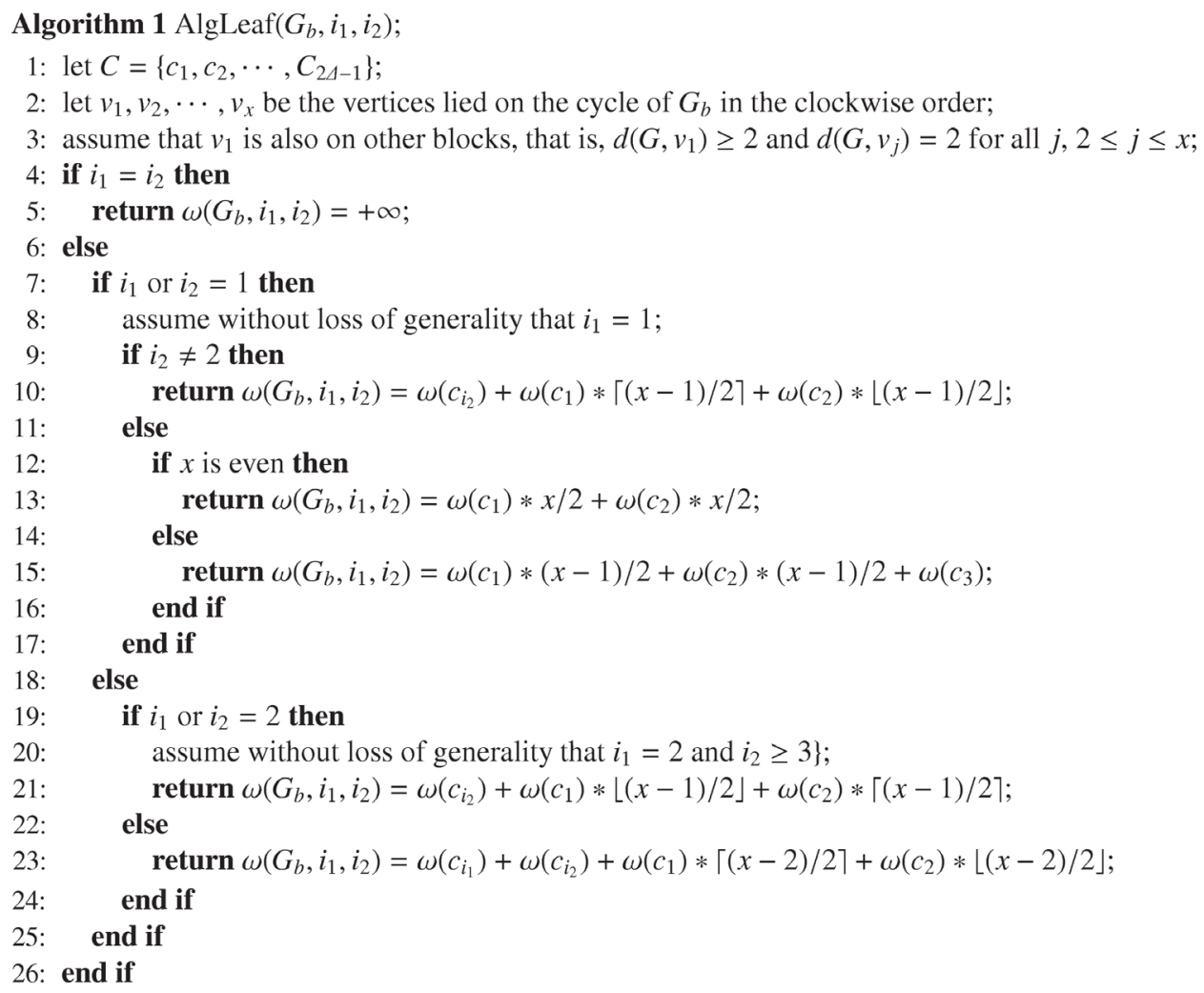




\subsubsection{The Node $b$ Is an Internal Node}

In order to compute $\omega\left(G_{b}, i_{1}, i_{2}\right)$ for each pair of indices $i_{1}$ and $i_{2}, \quad 1 \leq i_{1}, i_{2} \leq|C|$, we introduce a new parameter $\omega^{*}\left(B, v, i_{1}, i_{2}\right)$ defined as follows.

Let $B=\left\{b_{1}, b_{2}, \cdots\right\}$ be a set of blocks of $T$ such that all these blocks share exactly one vertex $v$ in $G$. For each pair of colors $c_{i_{1}}, c_{i_{2}} \in C$ we define

$$
\omega^{*}\left(B, v, i_{1}, i_{2}\right)=\min \left\{\omega(f) \mid f \text { is an edge-coloring of } G_{v} \text { and } c_{i_{1}}, c_{i_{2}} \in \operatorname{Miss}(f, v)\right\} \text {. }
$$

We show how to compute the all the values $\omega^{*}\left(B, v, i_{1}, i_{2}\right)$ from the $|B| \times|C|^{2}$ values $\omega\left(G_{b_{j}}, i_{1}, i_{2}\right)$, $1 \leq j \leq|B|$ and $1 \leq i_{1}, i_{2} \leq|C|$. The problem of computing $\omega^{*}\left(B, v, i_{1}, i_{2}\right)$ can be reduced to the minimum cost flow problem on a bipartite graph $K\left(i_{1}, i_{2}\right)$ as follows.

We first introduce $|B| \times|C|^{2}$ isolated vertices $v_{l_{1}, l_{2}}^{j}, 1 \leq j \leq|B|$ and $1 \leq l_{1}, l_{2} \leq|C|$. Then add $|C|$ vertices $v_{l}, 1 \leq l \leq|C|$, corresponding to colors $c_{l}$, and add a source $s$ and a sink $t$. Connect the source $s$ to all the $|C|$ vertices $v_{l}, 1 \leq l \leq|C|$, with capacity 1 and cost 0 . For each vertex $v_{l}, 1 \leq l \leq|C|$ and $l \notin\left\{i_{1}, i_{2}\right\}$, connect $v_{l}$ to all the vertices $v_{l_{1}, l_{2}}^{j}, 1 \leq j \leq \mid B$ and $1 \leq l_{1}, l_{2} \leq|C|$, satisfying $l_{1}=l$ or $l_{2}=l$ with capacity 1 and cost 0 . Finally, for each vertex $v_{l_{1}, l_{2}}^{j}, 1 \leq j \leq|B|$ and $1 \leq l_{1}, l_{2} \leq|C|$, connect $v_{l_{1}, l_{2}}^{j}$ to the sink $t$ with capacity 2 and cost $\omega\left(G_{b_{j}}, l_{1}, l_{2}\right)$. The minimum cost flow problem is to find a maximum flow from $s$ to $t$ with the sum of costs of edges on the flow. Clearly $\omega^{*}\left(B, v, i_{1}, i_{2}\right)$ is equal to the cost of the minimum cost maximum flow in $K\left(i_{1}, i_{2}\right)$.

The minimum cost maximum flow problem can be solved in time polynomial in the size of the graph [9] [10], and hence the value $\omega^{*}\left(B, v, i_{1}, i_{2}\right)$ for a pair of indices $i_{1}$ and $i_{2}, 1 \leq i_{1}, i_{2} \leq|C|$, can be computed in time polynomial in $|B|$ and $\mid C$ since $K\left(i_{1}, i_{2}\right)$ has at most $O\left(|B \| C|^{2}\right)$ vertices and edges. Therefore the $|C|^{2}$ values $\omega^{*}\left(B, v, i_{1}, i_{2}\right)$ for all pairs of indices $i_{1}$ and $i_{2}, 1 \leq i_{1}, i_{2} \leq|C|$, can be computed total in time polynomial in $|B|$ and $\mid C$.

We are now ready to compute $\omega\left(G_{b}, i_{1}, i_{2}\right)$. Since the block $b$ is either an edge or a cycle, we have the following two cases to consider.

Case 1: the block $b$ is an edge $e=(u, v)$.

Let $B=\left\{b_{1}, b_{2}, \cdots, b_{\mathrm{ch}(b)}\right\}$ be the set of blocks of the children of $b$ in $T$. Then all the blocks $b_{1}, b_{2}, \cdots, b_{\mathrm{ch}(b)}$ share exactly one vertex $v$ in $G$. In this case, clearly

$$
\omega\left(G_{b}, i_{1}, i_{2}\right)= \begin{cases}\omega^{*}\left(B, v, i_{1}, i_{1}\right) & \text { if } i_{1}=i_{2}, \\ +\infty & \text { if } i_{1} \neq i_{2} ;\end{cases}
$$

and it can be computed in time polynomial in the size of $G_{b}$ and $|C|$.

Case 2: the block $b$ is a cycle.

In this case, let $v_{1}, v_{2}, \cdots, v_{x}$ be the vertices lied on the cycle of $G_{b}$ in the clockwise order. Assume that $v_{1}$ is the vertex shared by the block $b$ and its parent block, and let $B\left(v_{j}\right), 2 \leq j \leq x$, be the set of blocks which shares $v_{j} ; B\left(v_{j}\right)=\varnothing$ if no such blocks exist. In order to compute $\omega\left(G_{b}, i_{1}, i_{2}\right)$ we define

$$
\omega_{1, j}^{*}\left(i_{1}, l_{j}\right)=\min _{1 \leq l_{2}, l_{3}, \cdots, l_{j-1} \leq C|C|}\left\{\sum_{2 \leq p \leq j} \omega^{*}\left(B\left(v_{p}\right), v_{p}, l_{p-1}, l_{p}\right)+\sum_{1 \leq p \leq j} \omega\left(c_{l_{p}}\right)\right\}
$$

for each $j, \quad 2 \leq j \leq x$, where $l_{1}=i_{1}$. Then clearly

$$
\omega\left(G_{b}, i_{1}, i_{2}\right)=\omega_{1, x}^{*}\left(i_{1}, i_{2}\right)
$$

Therefore it suffices to show how to compute $\omega_{1, j}^{*}\left(i_{1}, l_{j}\right)$ in polynomial time for each $j, 2 \leq j \leq x$, as follows.

By Equation (1) we have

$$
\begin{aligned}
\omega_{1, j+1}^{*}\left(i_{1}, l_{j+1}\right) & =\min _{1 \leq l_{2}, l_{3}, \cdots, l_{j} \leq C \mid}\left\{\sum_{2 \leq p \leq j+1} \omega^{*}\left(B\left(v_{p}\right), v_{p}, l_{p-1}, l_{p}\right)+\sum_{1 \leq p \leq j} \omega\left(c_{l_{p+1}}\right)\right\} \\
& =\min _{1 \leq l_{j} \leq C \mid}\left\{\omega_{1, j}^{*}\left(i_{1}, l_{j}\right)+\omega^{*}\left(B\left(v_{j+1}\right), v_{j+1}, l_{j}, l_{j+1}\right)+\omega\left(c_{l_{j+1}}\right)\right\},
\end{aligned}
$$


and hence $\omega_{1, j}^{*}\left(i_{1}, l_{j}\right)$ for all $j, 2 \leq j \leq x$, can be recursively computed total in time $O(x|C|)$ if all the values $\omega^{*}\left(B\left(v_{j}\right), v_{j}, l_{1}, l_{2}\right), \quad 1 \leq l_{1}, l_{2} \leq|C|$, are given. Since we have mentioned before that all the values $\omega^{*}\left(B\left(v_{j}\right), v_{j}, l_{1}, l_{2}\right)$ can be computed in time polynomial in $\left|B\left(v_{j}\right)\right|$ and $|C|$, one can compute all $\omega_{1, j}^{*}\left(i_{1}, l_{j}\right)$ and hence $\omega\left(G_{b}, i_{1}, i_{2}\right)$ total in time polynomial in $n\left(G_{b}\right)$ and $|C|$.

\section{Conclusion}

In this paper, we show that the cost edge-coloring problem for a cactus $G$ can be solved in polynomial time. It is still open to solve the problem in polynomial time for outerplanar graphs.

\section{Supported}

This work is partially supported by grants of the thousand talent plan of Zhejiang province.

\section{References}

[1] West, D.B. (2000) Introduction to Graph Theory. 2nd Edition, Prentice Hall, New Jersey.

[2] Hajiabolhassan, H., Mehrabadi, M.L. and Tusserkani, R. (2000) Minimal Coloring and Strength of Graphs. Discrete Mathematics, 215, 265-270. http://dx.doi.org/10.1016/S0012-365X(99)00319-2

[3] Mitchem, J., Morriss, P. and Schmeichel, E. (1997) On the Cost Chromatic Number of Outerplanar, Planar, and Line Graphs. Discussiones Mathematicae Graph Theory, 17, 229-241. http://dx.doi.org/10.7151/dmgt.1050

[4] Giaro, K. and Kubale, M. (2000) Edge-Chromatic Sum of Trees and Bounded Cyclicity Graphs. Information Processing Letters, 75, 65-69. http://dx.doi.org/10.1016/S0020-0190(00)00072-7

[5] Zhou, X. and Nishizeki, T. (2004) Algorithm for the Cost Edge-Coloring of Trees. J. Combinatorial Optimization, 8, 97-108. http://dx.doi.org/10.1023/B:JOCO.0000021940.40066.0c

[6] Coffman, E.G., Garey, M.R., Johnson, D.S. and LaPaugh, A.S. (1985) Scheduling File Transfers. SIAM J. Computing, 14, 744-780. http://dx.doi.org/10.1137/0214054

[7] Krawczyk, H. and Kubale, M. (1985) An Approximation Algorithm for Diagnostic Test Scheduling in Multicomputer Systems. IEEE Trans. Computers, 34, 869-872. http://dx.doi.org/10.1109/TC.1985.1676647

[8] Marx, D. (2009) Complexity Results for Minimum Sum Edge Coloring. Discrete Applied Mathematics, 157, 10341045. http://dx.doi.org/10.1016/j.dam.2008.04.002

[9] Goldberg, A.V. and Tarjan, R.E. (1987) Solving Minimum Cost Flow Problems by Successive Approximation. Proc. 19th ACM Symposium on the Theory of Computing, 7-18. http://dx.doi.org/10.1145/28395.28397

[10] Goldberg, A.V. and Tarjan, R.E. (1989) Finding Minimum-Cost Circulations by Canceling Negative Cycles. J. ACM, 36, 873-886. http://dx.doi.org/10.1145/76359.76368 